\title{
MicroRNA-200c Overexpression in Cancer- Associated Fibroblasts Reduces the Invasive Properties of Breast Cancer Cells: An Approach to Molecular Therapy
}

\author{
Nasim Shenavar \\ Isfahan University of Medical Sciences \\ Laleh Shariati \\ Isfahan University of Medical Sciences \\ Mohammad Reza Hakimian \\ Isfahan University of Medical Sciences
}

Shaghayegh Haghjooy Javanmard ( $\nabla$ sh_haghjoo@med.mui.ac.ir )

Isfahan University of Medical Sciences https://orcid.org/0000-0002-3853-5006

\section{Research Article}

Keywords: breast cancer, microenvironment, Fibroblast, miRNA, miR-200c, angiogenesis

Posted Date: March 17th, 2021

DOI: https://doi.org/10.21203/rs.3.rs-292182/v1

License: (c) (1) This work is licensed under a Creative Commons Attribution 4.0 International License.

Read Full License 


\section{Abstract}

\section{Background}

The most common malignancy is breast cancer, among women in the world. Triple-negative breast cancer (TNBC) is the most aggressive subtype of breast cancer. Cancer associated fibroblasts (CAFs) play a critical role to support tumor cells in all aspect of cancer development such as cell proliferation, migration and angiogenesis. MiRNAs are one of the regulatory molecules that regulate the genes contributing to cell growth, differentiation, migration and apoptosis. Based on other studies, miR-200c, as a tumor suppressor, has low expression levels in cancer associated fibroblasts. In this investigation, effect of miR-200c overexpression was evaluated on proliferation, migration and angiogenesis of TNBC cells.

\section{Methods}

The fibroblasts were isolated from normal and cancer breast tissue. MiR-200c expression was assessed using RT PCR in cancer associated fibroblasts (CAFs) and normal fibrobalasts (NFs) and then, were transfected using miR-200c. Finally, its effect on proliferation, migration and angiogenesis of TNBC cells were evaluated.

Results

Our results confirm that in presence of miR-200c transfected fibroblasts, the proliferation, migration and angiogenesis of cancer cells significantly decreased. This effect may be due to the reduction of growth factors provided by CAFs after miRNAs dysregulation.

Conclusion

These results suggest miR-200c act as an effective tumor suppressor in many aspects of TNBC cancer development and can be regarded as a potential therapeutic tool for breast cancer in the future.

\section{Key Points}

CAFs are known to play great role in breast cancer progress.

MiRNAs play an important role in the transformation of normal fibroblast to CAF

miRNA-200c overexpression decrease invasive features of cancer cells

\section{Background}

Breast cancer (BC) is the most common cancer and one of the main reasons of death among women (1 in 3 cases). Triple-negative breast cancer (TNBC) is the most aggressive subtype of BC, with earlier recurrence and poor prognosis. This subtype has a larger size tumor with faster growth and metastasis to 
farther organs. It is identified by lack of estrogen receptor (ER), progesterone receptor (PR), and hormone epidermal growth factor receptor 2 (HER2) $(1,2)$. Both environmental and genetic factors can interact to increase the risk of $\mathrm{BC}(3)$.

In recent years, the crucial role of tumor microenvironment (TME), also known as tumor stroma, has increasingly been elucidated (4). The modern concept is that cancer cells do manifest the disease only through collaborative interactions with their supporting stroma. TME plays a main role in many tumorigenic processes such as proliferative signaling, angiogenesis, invasion and metastasis that consequently lead to patient's death (5). TME consists of four elements: tumor vessels, immune cells, extracellular matrix (ECM) and fibroblasts, also known as tumor-associated fibroblasts (TAF) or cancer associated fibroblast ( CAF) $(4,6)$.

Fibroblasts are the most abundant cells in stroma contributed to many biologic processes such as secretion of ECM components, including growth factor and signal factors, wound healing and provide support for tissue cells in inflammation. Through these processes, quiescent fibroblasts undergo alternations, become active and convert to myofibroblast $(7,8)$. Similar to myofibroblast, CAFs support the tumor by providing suitable conditions under which the tumor can survive, grow, evade from the immune system and become metastatic (9). CAFs are the main source of factors such as TGF $\beta, E G F, a-$ SMA and VEGF, which participate in tumor progress, angiogenesis, migration and metastasis $(10,11)$.

The mechanism of fibroblast activation in TME is not completely understood. However, recent studies have confirmed that mutations and dysregulation of regulatory genes in stroma cells lead to the secretion of factors influencing tumor progression $(6,12)$. One of the regulatory genes are miRNAs that play an important role in the transformation of normal fibroblast to CAF (12). miRNAs are small noncoding RNAs that regulate the genes contributing to cell growth, differentiation, migration and apoptosis $(13,14)$. Based on recent reports, 11 groups of miRNAs are deregulated in CAF compared to normal fibroblast (NF) such as the miRNA-200 family, which cause secretion of supporting factors for tumor progression and development (15). Studies have revealed that miR-200c, as a tumor suppressor, has low expression levels in cancer associated fibroblasts that may prompt the invasion, migration and angiogenesis ability of cancer cells (16-18).

The present research was launched to assess the rate of proliferation, angiogenesis and migration of TNBC cell line after indirect co-culture with CAF and NF, overexpressing miR-200c, as compared to control cells.

\section{Methods}

\section{2-1 Isolation of fibroblasts from normal and cancer breast tissue}

Tumor and normal tissues specimens were obtained from stage II TNBC patients between the ages of 30 and 50 years undergoing lumpectomy and mastectomy at Ordibehesht surgery clinic, Isfahan, Iran. Written informed consent was obtained from each participants before surgery. The proposal was 
approved by the ethics committee of Isfahan University of Medical Sciences. The tumor samples were isolated from tumor zone (within tumor boundary) and normal samples isolated from normal zone (at least $10 \mathrm{~mm}$ distal from tumor boundary) and were taken to lab in Dulbecco's Modified Eagle's medium (DMEM)/F12 + 10\% FBS medium, sliced and digested with enzyme mixture contained $160 \mu \mathrm{g} / \mathrm{ml}$ of Collagenase IV and $25 \mu \mathrm{g} / \mathrm{ml}$ Hyaluronidase (19). Digested samples were incubated at $37^{\circ}$ with $5 \% \mathrm{CO}_{2}$ overnight and then cultured in DMEM/F12 + 10\% FBS for about a week.

\section{2-2 Cell lines}

BT-20. In 1958, BT-20 cell line derived from a 74-year-old Caucasian female with TNBC breast cancer, caused by invasive ductal carcinoma in mammary glands.

MDA-MB-231. This is an epithelial, human breast cancer cell line that is highly aggressive and invasive. MDA_MB-231 cell line was established from a pleural effusion of a 51-year-old Caucasian female with TNBC breast cancer.

HUVEC. The human umbilical vein endothelial cells, HUVEC, are endothelial like cells that are usually used for angiogenesis assays.

All the cell lines were purchased from Pasteur Institute of Iran, cultured in DMEM $+10 \%$ FBS medium and incubated at $37^{\circ}$ with $5 \% \mathrm{CO}_{2}$ for further assays.

\section{2-3 Immunocytochemistry (ICC)}

Alpha actin smooth muscle (a-SMA) is known as a specific marker widely expressed in fibroblast (20). To identify CAFs and NFs, the a-SMA (cat no. ab9654. Abcam) biomarker antibody were used and analyzed by ICC test based on the company instructions.

\section{2-4 Transfection of miR-200c-3p mimics and scramble}

MiR-200c-3p mimics (small, chemically modified double-stranded RNAs) (Cat No. MCH01501) and scramble (Cat No. MIR 7902) were purchased from abm and Mirus companies, respectively. Scramble is conjugated by FITC to measure the transfection rate. CAF and NF were transfected with mimic-miR-200c and scramble using the lipofectamine 2000 reagent (Invitrogen, Cat No. 13778030), according to the manufacturer's instructions.

\section{2-5 Real-time RT-PCR analysis}

Trizol reagent (Invitrogen, USA) use to RNA extraction, cDNA was synthesized and expression levels of mature has-mR-200c-3p was evaluated in NF, CAF, miR-200c transfected NF and miR-200c transfected CAF cells with ExiLENT SYBR ${ }^{\circledR}$ Green master mix kit (Exiqon, Denmark) using StepOne Plus ${ }^{\text {TM }}$ real-time PCR (Applied Biosystems, USA). Fold change was calculated using Livak method and ready to use 
primers were purchased from Exiqon and the SNORDs was used as a house keeping gene to normalize the data.

\section{2-6 Co-culture of isolated fibroblastand TNBC cell lines (BT-20 and MDA-MB-231)}

Study plan. There were five sets of triplicates in this study. The first set was TNBC breast cancer cells (BT20 and MDA-MC-231) that were not co-cultured with fibroblast which served as the negative control. The second set was co-cultured with un-transfected NF. The third set was co-cultured with scramble transfected NF. The fourth set was co-cultured with miR-200c transfected NF. The fifth set was cocultured with un-transfected CAF. The other set was co-cultured with scramble transfected CAF and the last set was co-cultured with transfected CAF. For co-culture, $3 \times 10^{3} \mathrm{CAF}$ and NF cells were plated in the upper well of transwell and $2 \times 10^{3}$ BT-20, MDA-MB-231 or HUVEC cells were seeded in lower well.

MTT assay. To investigate the proliferation of BT-20 and MDA-MB-231 cells after co-culturing with fibroblast, based on sets described above, MTT test was carried out. Two days after co-culturing, the upper wells were removed, then $50 \mu \mathrm{l}$ MTT solution ( $5 \mathrm{mg} / \mathrm{ml}$ ) (Sigma-Aldrich Co., USA) was added to

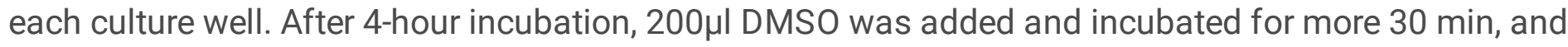
the absorbance was read at $570 \mathrm{~nm}$ by ELISA reader.

Wound healing assay. The directional cell migration of BT-20 and MDA-MB-231 cells was evaluated using wound healing assay after co-culturing with fibroblast, according to the groupings described above. BT-20 and MDA-MB-231 cells were plated. After $24 \mathrm{~h}$, the monolayer was scratched by a sterile pipette tip and the wells were obliterated of debris. Later, the co-culture was performed based on study plan section and allowed the cells to close the wound for 24,48 and $72 \mathrm{~h}$. Wound closure was measured with microscopic photography in 10 randomized field. The results were reported and analyzed by the imageJ software (version 1.52i).

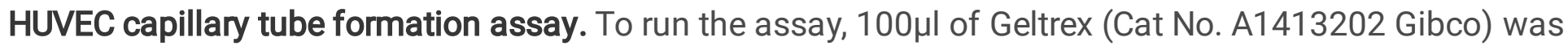
added to the lower plate of transwell to form a 3D vessel- like tubes, then $10{ }^{5} \mathrm{HUVEC}$ cells were seeded top of the Geltrex. Co-culture was performed based on study plan section and allowed the HUVEC cells to form tubes after about 24 and $48 \mathrm{~h}$. Image analysis was carried out using the Angioquant software (version v1.33). The size, length and number of branches were measured and reported.

\section{2-7 Statistical analysis}

The data were analyzed using one-way ANOVA and Mann-Whitney $U$ test. All results were shown as mean \pm SD and $P<0.05$ was considered as statistically significant.

\section{Results}


3-1 The fibroblasts isolated from normal and cancer breast tissues exhibit a-SMA marker. A combination of cells exists in isolated stromal cells from primary breast tissues that the main component of which are fibroblasts. The stromal fibroblasts isolated from normal and cancer breast tissues display features of NFs and CAFs. After 1 week, the cells gradually got out of the tissue sections (Fig 1Ag). Uniform fibroblasts initiate to grow after serial passage of primary cells (Fig 1B). The stromal fibroblasts separated from cancer and normal tissues expressed the activated myofibroblast marker a-SMA based on ICC test results (Fig 1C). The results of previous studies indicated that at low passages cultured in vitro, the isolated fibroblasts reserve features of CAFs and NFs (21).

\section{3-2 Transfection efficiency of fibroblast cells was about $75 \%$.}

Six hours after transfection of fibroblast with miR-200c and scramble, the rate of transfection was evaluated using fluorescence microscopy (Nikon Inverted Microscope-Japan) (Fig 2A,B) and flow cytometry (FACS Analysis-Becton, Dickinson and Company). The percentage of the cells containing scramble conjugated with FITC was about 78\% (Fig 2C,D).

\section{3-3 Real-time RT-PCR analysis.}

We examined the miR-200c level in NF, CAF, miR-200c transfected NF and miR-200c transfected CAF cells. The level of miR-200c in CAF was lower than that of NF, but this difference was not significant (Fig. 3A).

After $24 \mathrm{~h}$ of transfection, the level of miR-200c was elevated to 4.6 -fold in the miR-200c transfected NF cells compared to NF (Fig. 3B) and 3.5-fold in miR-200c transfected CAF cells compared to CAF (Fig. 3C). Therefore, this results robustly confirmed efficiency of transfection.

\section{3-4 MiR-200c-untransfected fibroblasts induced TNBC cell proliferation more than transfected fibroblasts.}

To investigate the effect of mir-200c level changes in CAF on cancer cells, the proliferation of BT-20 and MDA-MB-231 cells was assessed after co-culturing with NF, miR-200c- transfected NF, CAF, miR-200ctransfected CAF and without any co-culture.

Cell proliferation of MDA-MB-231 cells significantly increase compared to control cells after co-culturing with $\mathrm{NF}$ and $\mathrm{CAF}(\mathrm{P}=0.01$ and $\mathrm{P}=0.001$, respectively). This results confirm the supportive role of fibroblast in cancer cell proliferation. This rate is more significant for those cancer cells co-cultured with CAF than NF. On the other hand, proliferation of BT-20 and MDA-MB-231 cells significantly decreased after co-culturing with miR-200c-transfected NF $(P=0.000)$ and miR-200c-transfected CAF $(P=0.000)$ compared to un-transfected ones (Fig 4). This results confirm that miR-200c has tumor suppressor role for cancer cells. The scramble transfected CAF and NF did not have significant effect on TNBC cell proliferation compared to un-transfected CAF and NF $(P>0.05)$.

3-5 TNBC cell migration is significantly reduced after co-culturing with miR-200c transfected NF and miR$200 \mathrm{c}$ transfected CAF compared to untransfected ones. 
A wound healing assay was carried out under indirect co-culture conditions to confirm the effects of CAF, NF, miR-200c transfected CAF and NF stimulation on the BT-20 and MDA-MB-231 cell migration. The extent of the scratched zone narrowed when MDA-MB-231 and BT-20 cells were co-cultured with NFs, and this site was almost closed when MDA-MB-231 and BT-20 cells were co-cultured with CAFs within 72 hours $(P=0.000)$ (Fig 5)(The microscopic images related to $72 \mathrm{~h}$ after scratch are not shown). On the other hand, the size of the zone was broader when the cells were co-cultured with miR-200c transfected NF than untransfected NF $(P=0.005)$ and this change was more significant in the cells co-cultured with miR200c-transfected CAF than untransfected T-CAF $(P=0.000)$ (Fig 5). The scramble transfected CAF and NF did not have significant effect on TNBC cell migration compared to un-transfected CAF and NF $(P>0.05)$.

\section{3-6 Angiogenesis in HUVEC cells is significantly decreased after co-culturing with miR-200c transfected NF and miR-200c transfected CAF compared to the un-transfected ones.}

The anti-angiogenic effect of miR-200c overexpressing NF and CAF was evaluated after co-culturing with HUVEC tumor cells. We performed the in vitro HUVEC capillary tube formation assay. Figure 6 shows that HUVECs co-cultured with NF and CAF markedly produced capillary-like tubes compared with HUVECs cocultured with control cells $(P=0.05)$. In contrast, the angiogenesis notably suppressed in HUVEC cells after co-culturing with miR-200c transfected NF and CAF ( $P=0.000)$. Hence, poor vascularization involving small size, length and few branches of tubes was detected. This results confirm the suppressing role of miR-200c on angiogenesis stimulating behavior. The scramble transfected CAF and NF did not have significant effect on HUVEC cell angiogenesis compared to un-transfected CAF and NF ( $P>0.05)$.

\section{Discussion}

Tumor microenvironment, act as a supporting compartment in cancer process by providing a dynamic and physiologic niche for tumor. CAF, as a main functional cell in TME, produces and secretes growth factors, angiogenic factors which are a key player in EMT process and chemokines and cytokines that help migration and invasion of tumor leading to metastasis (22). Moreover, CAFs facilitate tumor cell movement and migration due to remodeling the ECM structure via changes in E-cadherin production, cellcell and cell-ECM junctions $(22,23)$.

Previous studies have shown that CAFs can provide a perfect niche for the tumor to develop and grow (24). Similar lines of evidence were found in our study and the supportive role of CAFs was confirmed, they can trigger the early steps of tumor development and cell proliferation. Our observation showed that cancer cells grow better and faster in the presence of CAFs compared to NF. This may be due to of quiescent nature of normal fibroblasts.

The co-culture of NF/CAF with BT-20 and MDA-MB-231 cells revealed that migration rate of cancer cells was increased compared to when cancer cells were grown alone. However, the increase was more pronounced in the cancer cells co-cultured with CAFs than NFs. After about $72 \mathrm{~h}$, the wound had not completely healed in in the cancer cells co-cultured with NF, while it completely healed when co-cultured with CAF. 
Angiogenesis in tumor is a complex process that depends on several angiogenic factors such as VEGF (25). The present study demonstrates that angiogenesis is improved in the vicinity of the CAF, where the secretion of VEGF is high, as also confirmed in the past (26).

Generally, the manipulation of gene regulation in cancer microenvironment is an attractive therapeutic strategy for reducing proliferation, migration and angiogenesis. Fibroblast components are produced under control of genes, which are, in turn, regulated by miRNAs. Observations have identified about 11 dysregulated miRNAs in CAFs, whose target genes mostly participate in proliferation, angiogenesis, cell differentiation, migration, secretion and cell adhesion (15). In this study, we focused on miR-200c, one of the main member of miR-200 family, which is down-regulated in CAF.

MiR-200 family target many genes such as MSN, FN1, MARCKS, QKI, FGD1, LOX, KDR, PAG1,ZEB and SIP1 which have an important role in TGF $\beta$-induced EMT process, cell adherences, migration and invasion (2729). In addition, these family members are known to be tumor suppressor and the lack of these molecules could lead to aggressive phenotype in many cancers such as gastric (30) glioma (31) lung (32) and breast (33). Some evidence demonstrate that miR-200 family are downregulated in breast cancer CAFs compared to NF (34).

There is much evidence regarding the regulatory effects of miR-200c on the nature of cancer (35). miR$200 \mathrm{c}$ regulates insulin receptor substrate 1 (IRS1) post transcriptionally and its over expression may be retard to the growth of prostate cancer cells (36). Likewise, overexpression of miR-200c in melanoma cells remarkably reduce the proliferation (37). Some studies indicated miR-200c overexpression in breast cancer cells, like MDA-MB-231, can reduce the EMT and migration $(38,39)$. Our observation revealed that miR-200c transfected CAFs and NFs co-cultured with TNBC basal and luminal breast cancer notably reduced growth and proliferation of tumor. Accordingly, the features of cancer cells which were cocultured with CAFs showed a significant difference compared to the group co-cultured with miR-200c transfected CAFs. However, this gap was less significant between the group co-cultured with NF and that co-cultured with miR-200c transfected NF.

Mir-200 family cooperate in tumor invasion and metastasis through inhibition of ZEB1 and ZEB2 gene expression (40). MiR-200c plays also an effective part in cancer metastasis via interference in TGF $\beta$ pathway (41). Here, we observed that transfection of CAFs with miR-200c can dramatically reduce breast cancer cell migration and invasion ability. Furthermore, overexpression of miR-200c in NF may also lead to deceased malignant cells invasion, nevertheless its effect is negligible. According to the wound assay, the scratch in the CAF co-cultured group was healed in a very short time while, the miR-200c-transfected CAF co-cultured group needed a much longer time for healing. The same pattern was observed in the NFs, but with a milder slope.

Increasing studies has revealed the therapeutic role of miR-200c on tumor angiogenesis. Accordingly, miR-200c can inhibit the tumor vacuolization (42). Our observation showed that transfection CAFs with miR-200c significantly decreased the angiogenesis in HUVEC cells. As it was found in angiogenesis test, CAF co-cultured group formed more tubes which were bigger in size and more branched than the control 
group. In addition, with a little difference, the NF co-cultured group showed the same results. Other

findings testify the supportive role of some CAF biomarkers such as miR-200c (43).

\section{Conclusion}

These results confirm that transfection of CAFs with miR-200c may limit variable aspects of breast cancer development such as growth, migration and angiogenesis. In this way, miR-200c can be regarded as a potential therapeutic tool for breast cancer in the future.

\section{Abbreviations}

Breast cancer $(\mathrm{BC})$

Triple-negative breast cancer (TNBC)

estrogen receptor (ER)

progesterone receptor (PR)

hormone epidermal growth factor receptor 2 (HER2)

tumor microenvironment (TME)

extracellular matrix $(\mathrm{ECM})$

tumor-associated fibroblasts (TAF)

cancer associated fibroblast ( CAF)

normal fibroblast (NF)

Alpha actin smooth muscle (a-SMA)

Immunocytochemistry (ICC)

\section{Declarations}

Ethics approval and consent to participate: This article does not contain any studies with human participants or animals performed by any of the authors.

Consent for publication: Not applicable

Availability of data and materials section: Not applicable 
Funding: This study was funded by Isfahan University of Medical Sciences deputy of research (grant number 396159). The funding body was used to buy consumables materials.

Author contribution statement: The authors, NS and LS contributed in practical work, acquisition of data, or analysis and interpretation of data.

The author, MRH contributed in sample preparing. The author, SHJ contributed in conception and design, or acquisition of data, or analysis and interpretation of data

Acknowledgement: We are deeply grateful to Isfahan University of Medical Sciences deputy of research due to their support (Grant No. 396159).

\section{References}

1. Dent R, Trudeau M, Pritchard KI, Hanna WM, Kahn HK, Sawka CA, et al. Triple-negative breast cancer: clinical features and patterns of recurrence. Clinical cancer research. 2007;13(15):4429-34.

2. Haffty BG, Yang Q, Reiss M, Kearney T, Higgins SA, Weidhaas J, et al. Locoregional relapse and distant metastasis in conservatively managed triple negative early-stage breast cancer. Journal of clinical oncology. 2006;24(36):5652-7.

3. Key TJ, Verkasalo PK, Banks E. Epidemiology of breast cancer. The lancet oncology. 2001;2(3):13340.

4. Hui L, Chen Y. Tumor microenvironment: Sanctuary of the devil. Cancer letters. 2015;368(1):7-13.

5. Hanahan D, Coussens LM. Accessories to the crime: functions of cells recruited to the tumor microenvironment. Cancer cell. 2012;21(3):309-22.

6. Hall B, Dembinski J, Sasser AK, Studeny M, Andreeff M, Marini F. Mesenchymal stem cells in cancer: tumor-associated fibroblasts and cell-based delivery vehicles. International journal of hematology. 2007;86(1):8-16.

7. Cirri P, Chiarugi P. Cancer-associated-fibroblasts and tumour cells: a diabolic liaison driving cancer progression. Cancer and Metastasis Reviews. 2012;31(1-2):195-208.

8. Bhowmick NA, Neilson EG, Moses HL. Stromal fibroblasts in cancer initiation and progression. Nature. 2004;432(7015):332.

9. Micke P. Tumour-stroma interaction: cancer-associated fibroblasts as novel targets in anti-cancer therapy? Lung cancer. 2004;45:S163-S75.

10. Aboussekhra A. Role of cancer-associated fibroblasts in breast cancer development and prognosis. International Journal of Developmental Biology. 2011;55(7-8-9):841-9.

11. Östman A, Augsten M. Cancer-associated fibroblasts and tumor growth-bystanders turning into key players. Current opinion in genetics \& development. 2009;19(1):67-73.

12. Chou J, Shahi P, Werb Z. microRNA-mediated regulation of the tumor microenvironment. Cell cycle. 2013;12(20):3262-71. 
13. Chou J, Werb Z. MicroRNAs play a big role in regulating ovarian cancer-associated fibroblasts and the tumor microenvironment. Cancer discovery. 2012;2(12):1078-80.

14. Iorio MV, Ferracin M, Liu C-G, Veronese A, Spizzo R, Sabbioni S, et al. MicroRNA gene expression deregulation in human breast cancer. Cancer research. 2005;65(16):7065-70.

15. Zhao L, Sun Y, Hou Y, Peng Q, Wang L, Luo H, et al. MiRNA expression analysis of cancer-associated fibroblasts and normal fibroblasts in breast cancer. The international journal of biochemistry \& cell biology. 2012;44(11):2051-9.

16. Li J, Tan Q, Yan M, Liu L, Lin H, Zhao F, et al. miRNA-200c inhibits invasion and metastasis of human non-small cell lung cancer by directly targeting ubiquitin specific peptidase 25 . Molecular cancer. 2014;13(1):166.

17. Tang $X$, Hou Y, Yang G, Wang X, Tang S, Du Y, et al. Stromal miR-200s contribute to breast cancer cell invasion through CAF activation and ECM remodeling. Cell death and differentiation. 2016;23(1):132.

18. Li L, Li B, Chen D, Liu L, Huang C, Lu Z, et al. miR-139 and miR-200c regulate pancreatic cancer endothelial cell migration and angiogenesis. Oncology reports. 2015;34(1):51-8.

19. Yu Y, Xiao CH, Tan LD, Wang QS, Li XQ, Feng YM. Cancer-associated fibroblasts induce epithelialmesenchymal transition of breast cancer cells through paracrine TGF-beta signalling. British journal of cancer. 2014;110(3):724-32.

20. Madar S, Goldstein I, Rotter V. 'Cancer associated fibroblasts'-more than meets the eye. Trends in molecular medicine. 2013;19(8):447-53.

21. Masur S, Dewal H, Dinh T, Erenburg I, Petridou S. Myofibroblasts differentiate from fibroblasts when plated at low density. Proceedings of the National Academy of Sciences. 1996;93(9):4219-23.

22. Swartz MA, lida N, Roberts EW, Sangaletti S, Wong MH, Yull FE, et al. Tumor microenvironment complexity: emerging roles in cancer therapy. Cancer research. 2012:canres. 0122.2012.

23. Balkwill FR, Capasso M, Hagemann T. The tumor microenvironment at a glance. The Company of Biologists Ltd; 2012.

24. Karagiannis GS, Poutahidis T, Erdman SE, Kirsch R, Riddell RH, Diamandis EP. Cancer-associated fibroblasts drive the progression of metastasis through both paracrine and mechanical pressure on cancer tissue. Molecular cancer research. 2012;10(11):1403-18.

25. Lazăr D, Raica M, Sporea I, Tăban S, Goldiş A, Cornianu M. Tumor angiogenesis in gastric cancer. Rom J Morphol Embryol. 2006;47(1):5-13.

26. Pietras K, Östman A. Hallmarks of cancer: interactions with the tumor stroma. Experimental cell research. 2010;316(8):1324-31.

27. Sundararajan V, Gengenbacher N, Stemmler MP, Kleemann JA, Brabletz T, Brabletz S. The ZEB1/miR200c feedback loop regulates invasion via actin interacting proteins MYLK and TKS5. Oncotarget. 2015;6(29):27083.

28. Gregory PA, Bert AG, Paterson EL, Barry SC, Tsykin A, Farshid G, et al. The miR-200 family and miR205 regulate epithelial to mesenchymal transition by targeting ZEB1 and SIP1. Nature cell biology. 
2008;10(5):593.

29. Schoepp M, Ströse AJ, Haier J. MicroRNAs in the Communication between Cancer Associated Fibroblasts (CAF) and Cancer Cells. 2017.

30. Kurashige J, Kamohara H, Watanabe M, Hiyoshi Y, Iwatsuki M, Tanaka Y, et al. MicroRNA-200b regulates cell proliferation, invasion, and migration by directly targeting ZEB2 in gastric carcinoma. Annals of surgical oncology. 2012;19(3):656-64.

31. Guo E, Wang Z, Wang S. MiR-200c and miR-141 inhibit ZEB1 synergistically and suppress glioma cell growth and migration. Eur Rev Med Pharmacol Sci. 2016;20(16):3385-91.

32. Bai T, Dong D-S, Pei L. Synergistic antitumor activity of resveratrol and miR-200c in human lung cancer. Oncology Reports. 2014;31(5):2293-7.

33. Ceppi P, Mudduluru G, Kumarswamy R, Rapa I, Scagliotti GV, Papotti M, et al. Loss of miR-200c expression induces an aggressive, invasive, and chemoresistant phenotype in non-small cell lung cancer. Molecular cancer research. 2010:1541-7786. MCR-10-0052.

34. Sun Y, Yang D, Xi L, Chen Y, Fu L, Sun K, et al. Primed atypical ductal hyperplasia-associated fibroblasts promote cell growth and polarity changes of transformed epithelium-like breast cancer MCF-7 cells via miR-200b/c-IKKß signaling. Cell death \& disease. 2018;9(2):122.

35. Shimono Y, Zabala M, Cho RW, Lobo N, Dalerba P, Qian D, et al. Downregulation of miRNA-200c links breast cancer stem cells with normal stem cells. cell. 2009;138(3):592-603.

36. Su W, Xu M, Chen X, Nie L, Chen N, Gong J, et al. MiR200c targets IRS1 and suppresses prostate cancer cell growth. The Prostate. 2015;75(8):855-62.

37. Liu S, Tetzlaff MT, Cui R, Xu X. miR-200c inhibits melanoma progression and drug resistance through down-regulation of BMI-1. The American journal of pathology. 2012;181(5):1823-35.

38. Ast V, Kordaß T, Oswald M, Kolte A, Eisel D, Osen W, et al. MiR-192, miR-200c and miR-17 are fibroblast-mediated inhibitors of colorectal cancer invasion. Oncotarget. 2018;9(85):35559.

39. Ingthorsson S, Briem E, Bergthorsson JT, Gudjonsson T. Epithelial plasticity during human breast morphogenesis and cancer progression. Journal of mammary gland biology and neoplasia. 2016;21(3-4):139-48.

40. Li X, Roslan S, Johnstone C, Wright J, Bracken C, Anderson M, et al. MiR-200 can repress breast cancer metastasis through ZEB1-independent but moesin-dependent pathways. Oncogene. 2014;33(31):4077.

41. Chen W, Zhou S, Mao L, Zhang H, Sun D, Zhang J, et al. Crosstalk between TGF- $\beta$ signaling and miRNAs in breast cancer metastasis. Tumor Biology. 2016;37(8):10011-9.

42. D'Ippolito E, Plantamura I, Bongiovanni L, Casalini P, Baroni S, Piovan C, et al. miR-9 and miR-200 regulate PDGFR $\beta$-mediated endothelial differentiation of tumor cells in triple-negative breast cancer. Cancer research. 2016.

43. Rupaimoole R, Slack FJ. MicroRNA therapeutics: towards a new era for the management of cancer and other diseases. Nature reviews Drug discovery. 2017;16(3):203. 


\section{Figures}
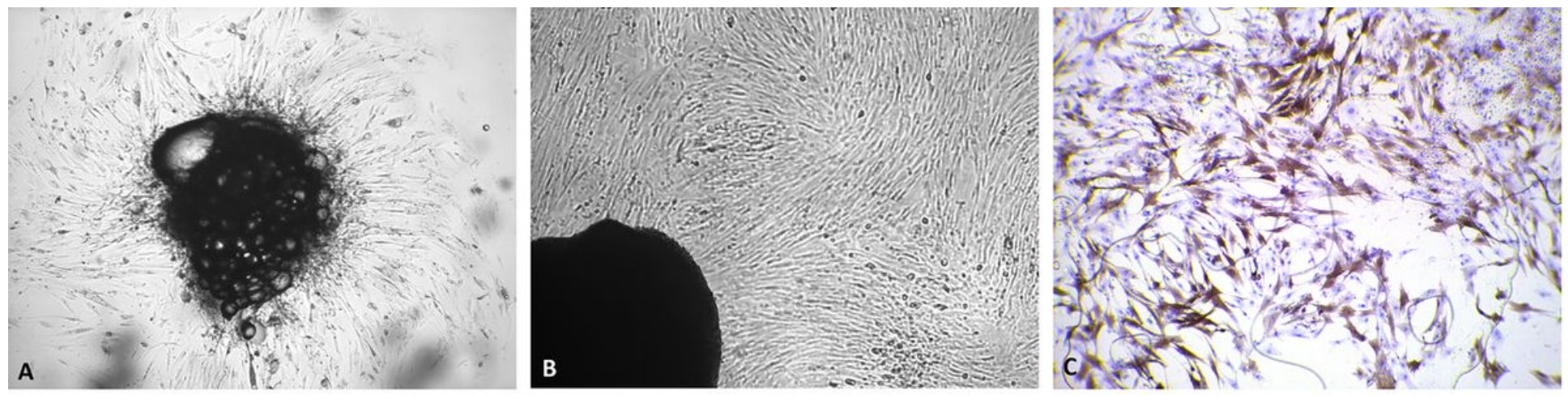

\section{Figure 1}

Fibroblasts isolation. A. Fibroblasts gradually got out of the tissue sections at day 7. B. Uniform fibroblasts at day 28. C. ICC staining for a-SMA to confirm the isolated fibroblast.
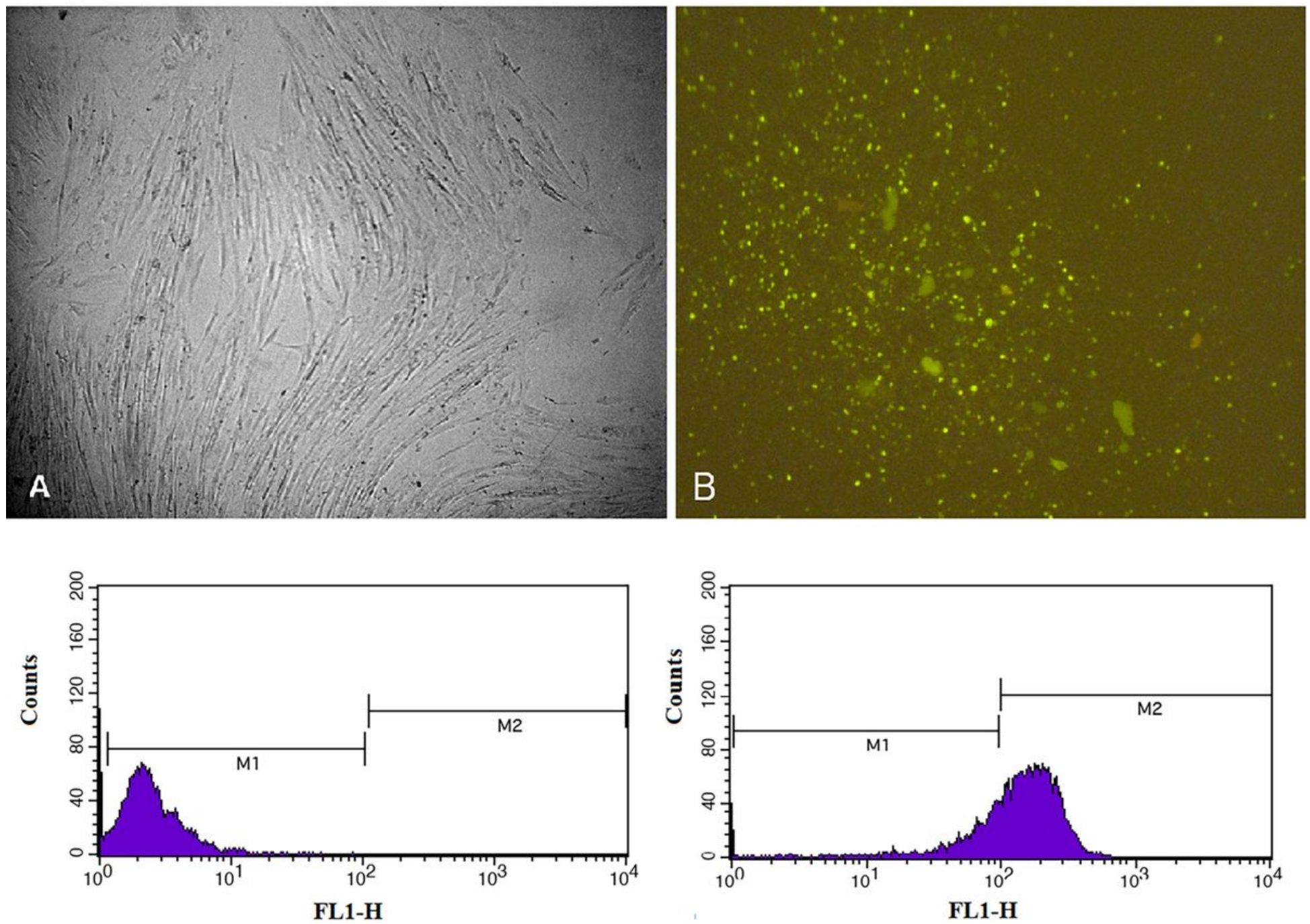

C

D

Figure 2 
Evaluation of transfection efficiency in fibroblast cells using fluorescence microscopy (A \&B) and Flow cytometry (C\&D). Fibroblast cells were transfected with scramble conjugated with FITC and assessed $5 \mathrm{~h}$ after transfection. A. The cells under visible light, B. The cells under blue $(470 \mathrm{~nm})$ band pass filter of fluorescence microscopy. C. Fibroblast cells without transfection have no FITC's emission. D. Fibroblast cells after transfection have 78\% FITC's emission.

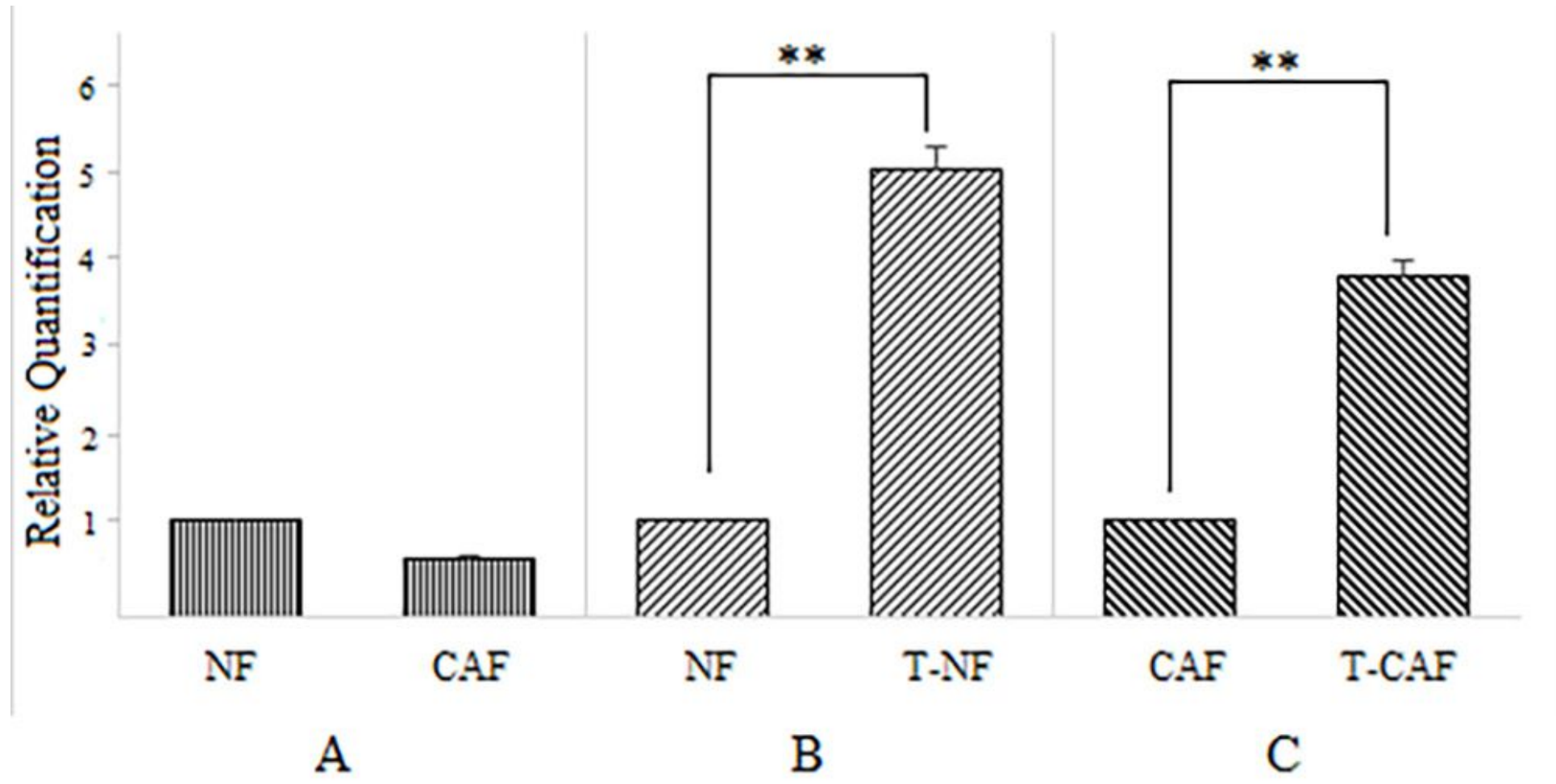

Figure 3

Relative quantification of miR-200c expression. A. The expression of miR-200c was compared between miR-200c transfected NF and NF cells by quantification comparative Ct Real time PCR. The level of miR200c in CAF was lower than that of NF, but this difference was not significant. B. The miR-200c transfected NF cells significantly increased miR-200c expression vs. NF. C. The miR-200c transfected CAF cells significantly increased miR-200c expression vs. CAF. ${ }^{*} P<0.05$, all results are means \pm SD from three independent experiments. Statistical significance was calculated using the Mann-Whitney $U$ test. 


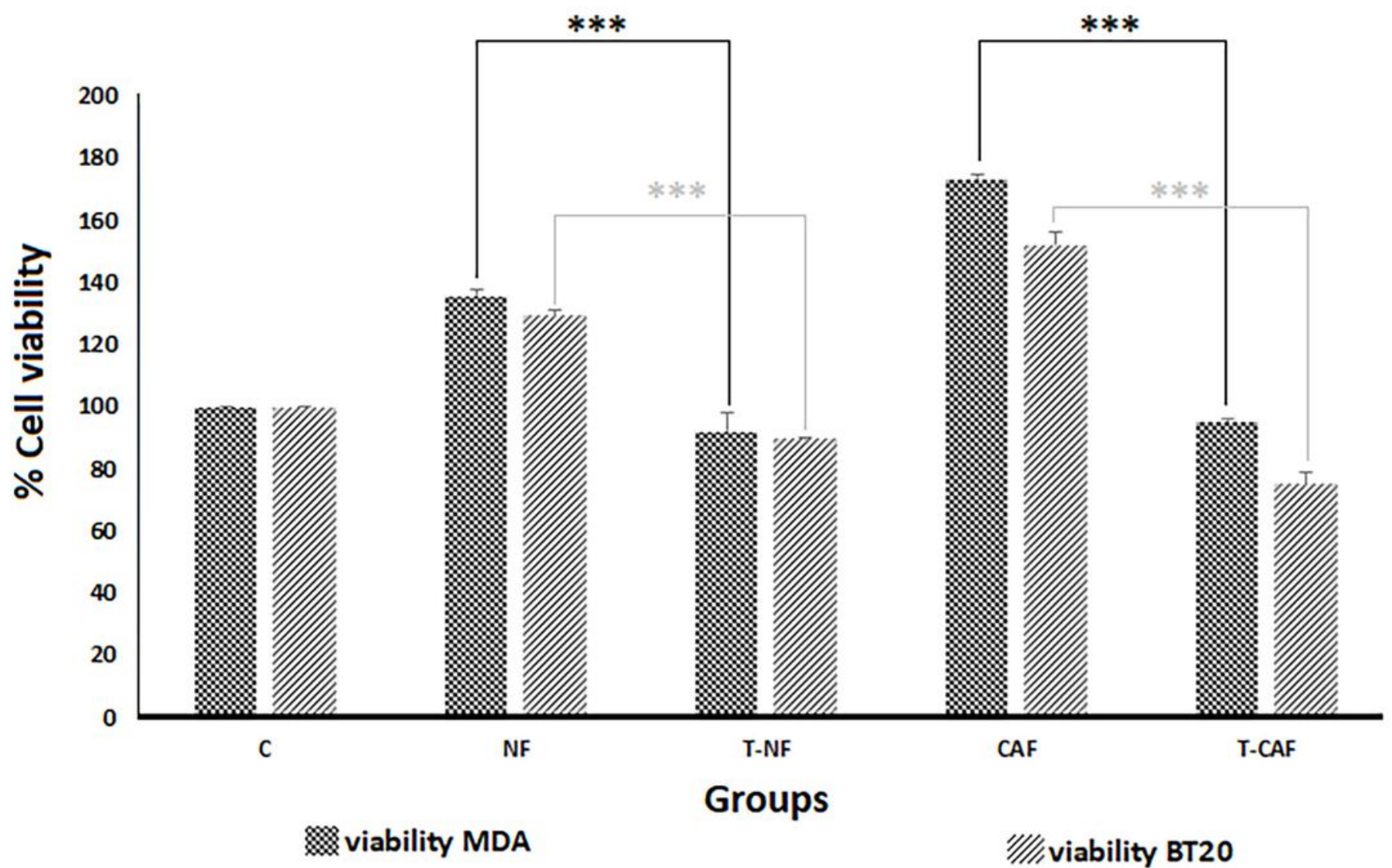

Figure 4

Cell viability in TNBC cells co-cultured with miR-200c un-transfected and transfected fibroblasts and the control group. MiR-200c-untransfected fibroblasts have inhibitory effects on the proliferation rate of BT20 and MDA-MB-231 cells. Points represent means of three experiments; bars represent SD, (*** $P=0.000)$ (C: Control, NF: Normal fibroblast, T-NF: miR-200c transfected- normal fibroblast, CAF: Cancer associated fibroblast, T-CAF: miR-200c transfected- Cancer associated fibroblast). 


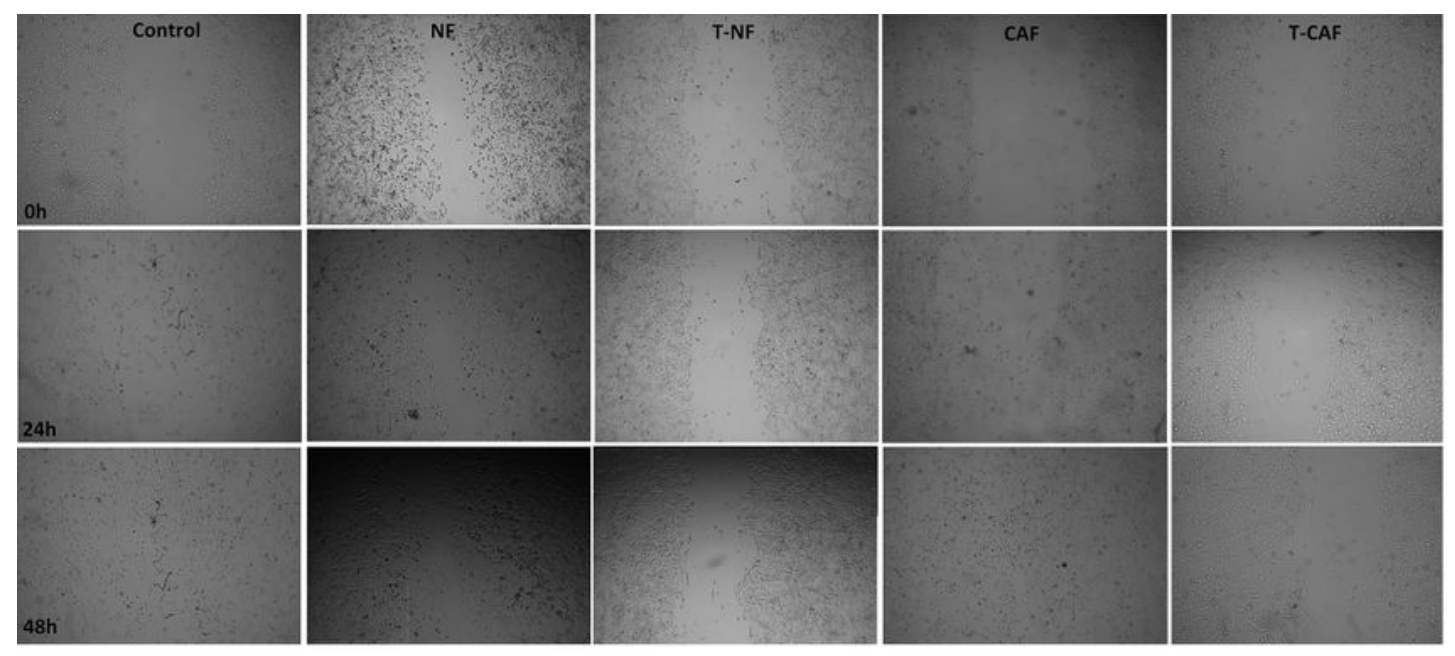

A
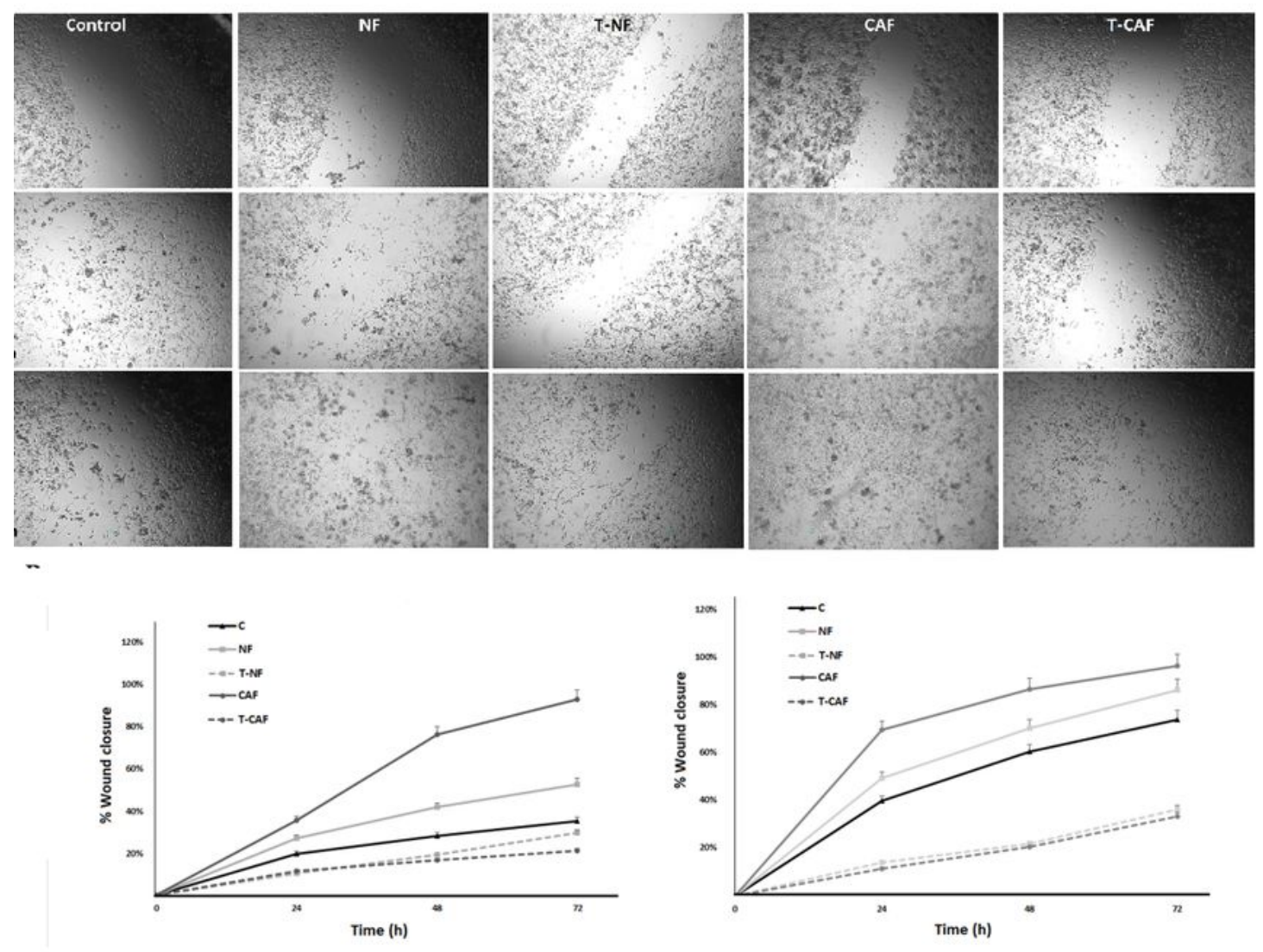

$\begin{array}{llll} & \mathbf{2 4 h} & \mathbf{4 8 h} & \mathbf{7 2 h} \\ \text { Control } & 19.5 & 21.5 & 35 \\ \text { NF } & 29 & 41.5 & 52.5 \\ \text { T-NF } & 8.5 & 19.5 & 31 \\ \text { CAF } & 35.5 & 76 & 92.5 \\ \text { T-CAF } & 11 & 16.5 & 21\end{array}$

$\begin{array}{llll} & \mathbf{2 4 h} & \mathbf{4 8 h} & \mathbf{7 2 h} \\ \text { Control } & 40.5 & 59.8 & 74.6 \\ \text { NF } & 50.9 & 68.8 & 87.6 \\ \text { T-NF } & 15 & 20.9 & 32.9 \\ \text { CAF } & 71.9 & 89 & 97.4 \\ \text { T-CAF } & 12.2 & 19.7 & 31.6\end{array}$

\section{Figure 5}

In vitro wound healing assay to determine migration capacity. A. MDA-MB-231 cells co-cultured with NF, T$\mathrm{NF}, \mathrm{CAF}, \mathrm{T}-\mathrm{CAF}$, then migration was assessed using a wound healing assy. B. BT-20 cells co-cultured with NF, T-NF, CAF, T-CAF, then migration was assessed using a wound healing assy. C. Quantification of open wound area and computing wound closure for MDA-MB-231 cells co-cultured with NF, T-NF, CAF, T-CAF. D. Quantification of open wound area and computing wound closure for BT-20 cells co-cultured with NF, T- 
NF, CAF, T-CAF. Points represent means of three experiments; bars represent $S D$, ( $\left.{ }^{\star \star *} \mathrm{P}=0.000,{ }^{\star *} \mathrm{P}=.0005\right)$ (C: Control, NF: Normal fibroblast, T-NF: miR-200c transfected- normal fibroblast, CAF: Cancer associated fibroblast, T-CAF: miR-200c transfected- Cancer associated fibroblast).
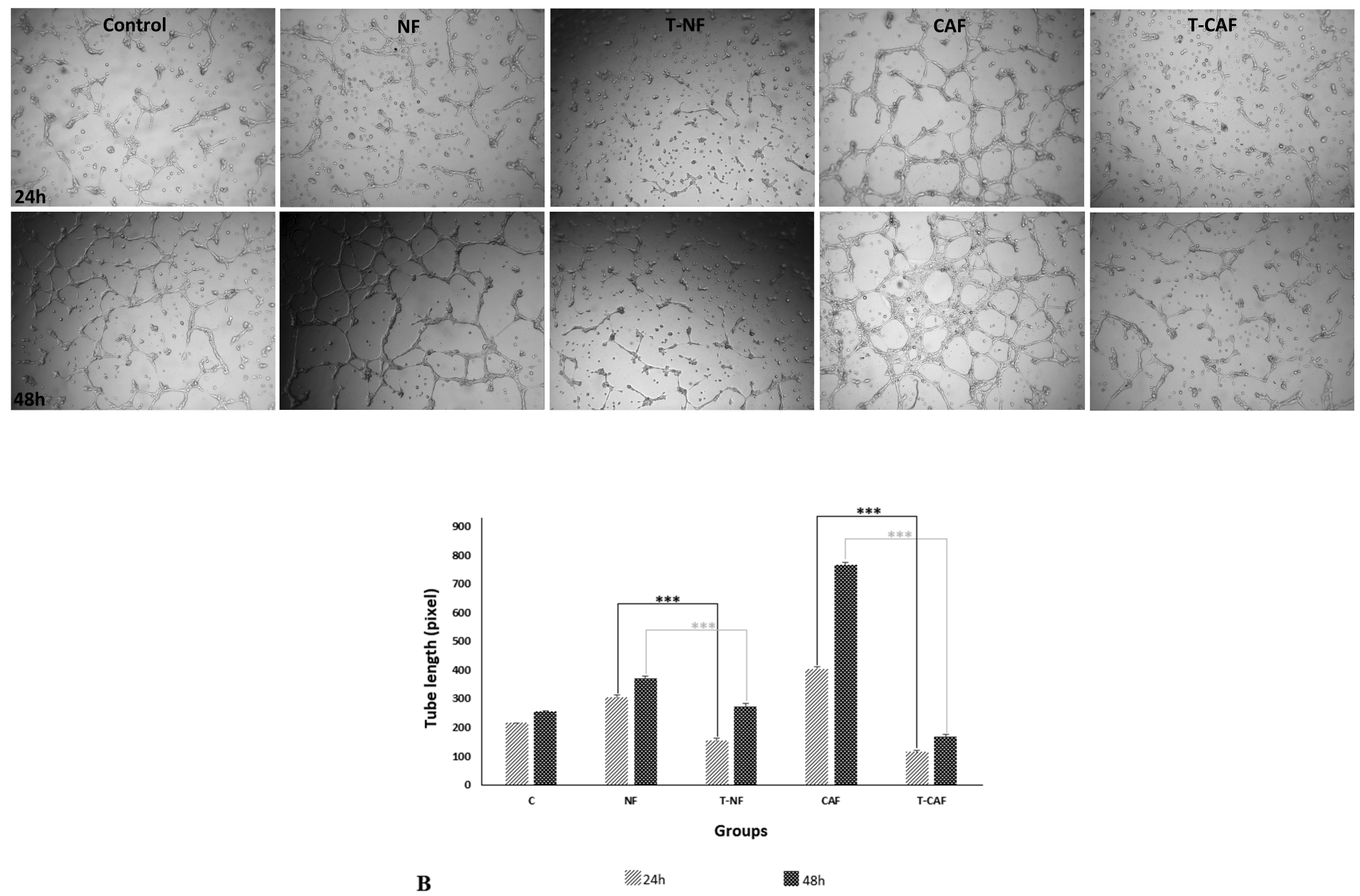

\section{Figure 6}

HUVEC capillary tube formation assay. A. The angiogenesis rate in HUVEC cells in vicinity of NF, T-NF, CAF and T-CAF after $24 \mathrm{~h}$ and $48 \mathrm{~h}$. B. Comparison of tube length rate among groups. Points represent means of three experiments; bars represent $S D,\left({ }^{* \star *} \mathrm{P}=0.000\right)$ (C: Control, NF: Normal fibroblast, T-NF: miR-200c transfected- normal fibroblast, CAF: Cancer associated fibroblast, T-CAF: miR-200c transfected-Cancer associated fibroblast). 